\title{
Refluxo gastroesofágico em cadelas submetidas à ovário-histerectomia convencional ou videoassistida
}

[Gastroesophageal reflux in dogs undergoing convencional or video-assisted ovariohysterectomy]

\author{
H.F. Hartmann ${ }^{1}$, J.P.S. Feranti ${ }^{1}$, M.T. Oliveira $^{1}$, M.T. Linhares $^{1}$, L.F.D. Correa ${ }^{1}$, G.P. Coradini ${ }^{1}$, \\ S.L. Abati ${ }^{1}$, M.V. Brun ${ }^{1,2} *$ \\ ${ }^{1}$ Universidade Federal de Santa Maria - Santa Maria, RS \\ ${ }^{2}$ Bolsista do CNPq - Brasil (308019/2015-6; 200346/2017-2)
}

\begin{abstract}
RESUMO
A doença do refluxo gastroesofágico decorre do fluxo de conteúdo gastroduodenal para o esôfago e/ou órgãos adjacentes, o que leva à ampla gama de sinais e implicações clínicas. A incidência de refluxo gastroesofágico transoperatório em caninos é desconhecida. O objetivo deste trabalho foi, por meio da endoscopia flexível, avaliar a presença e quantificar o refluxo gastroesofágico em cadelas submetidas à ovário-histerectomia por duas abordagens cirúrgicas (convencional e videoassistida com dois portais), pré-medicadas com morfina. Cem por cento das cadelas submetidas à ovário-histerectomia videoassistida e 30\% das cadelas submetidas à ovário-histerectomia convencional apresentaram algum grau de refluxo. A intensidade dos refluxos foi maior nas cadelas submetidas ao procedimento minimamente invasivo, visto que elas (10 entre 10 animais) apresentaram, no mínimo, um refluxo classificado em R4, enquanto as outras (três entre 10) apresentaram, no máximo, refluxos em grau R3, de acordo com a escala planejada para esta pesquisa. Concluiu-se que os procedimentos laparoscópicos sob o protocolo anestésico utilizado promovem mais refluxo gastroesofágico que os convencionais.
\end{abstract}

Palavras-chave: endoscopia, regurgitação transoperatória, cirurgia, videocirurgia, canino

\begin{abstract}
Gastroesophageal reflux disease occurs when gastric or duodenal contents flow back into the esophagus by retroperistalsis, which leads to several signs and clinical implications. The incidence of intraoperative gastroesophageal reflux in canines is unknown. The aim of this study was, using flexible endoscopy, to assess the presence and quantify gastroesophageal reflux in bitches undergoing ovariohysterectomy by two surgical approaches (conventional and video-assisted by two portals-access), pre-medicated with morphine. $100 \%$ of dogs submitted to video-assisted and 30\% of dogs submitted to conventional ovariohysterectomy presented some reflux degree. Reflux intensity was higher in dogs submitted to the minimally invasive procedure, since they (10 out of 10 animals) had at least one reflux classified in $R 4$ while the others (three out of 10) had a maximum reflux in degree R3 according to the planned scale for this research. The laparoscopic procedures under the used anesthetic protocol promote more gastroesophageal reflux than the conventional ones.
\end{abstract}

Keywords: endoscopy, intraoperative regurgitation, surgery, videosurgery, canine

\section{INTRODUÇÃO}

A doença do refluxo gastroesofágico (DRGE) em humanos é considerada atualmente uma das afecções crônicas mais importantes na prática médica, com elevada morbidade, prejuízo na

Recebido em 24 de agosto de 2016

Aceito em 15 de março de 2017

*Autor para correspondência (corresponding author)

E-mail: mauriciovelosobrun@hotmail.com qualidade de vida, frequentes recidivas e necessidade do uso continuado de medicação (Barczinski e Moraes-Filho, 2006). Está associada ao fluxo de conteúdo gastroduodenal para o esôfago e/ou órgãos adjacentes, o que leva à ampla gama de sinais clínicos (Norton e Pena, 2000; Barczinski e Moraes-Filho, 2006; Carvalhaes et al., 2011; Abrahão Jr., 2014). 
Sabe-se que a aspiração pulmonar de conteúdos gástricos é uma das principais causas anestésicas de morbidade e mortalidade (Nogueira et al., 2003). A incidência de pneumonia aspirativa pós-anestesia resultante de refluxo gastroesofágico em cães - 0,17\% (Ovbey et al., 2014) - é muito maior do que a relatada em humanos - 0,014 a 0,05\% (Janda e Scheeren, 2006). De outra forma, a pneumonia aspirativa pós-operatória eleva significantemente as taxas de morbidade e mortalidade nas unidades de tratamento intensivo em hospitais humanos (D'Haese et al., 2013). Dois eventos relacionados à anestesia foram associados ao desenvolvimento de pneumonia aspirativa em pequenos animais: a regurgitação e a administração de hidromorfona na indução (Ovbey et al., 2014).

Uma das desordens gastrointestinais mais comuns nos humanos é a doença do refluxo gastroesofágico, que causa azia, regurgitação e esofagite. Em cães, a verdadeira incidência dessa desordem é desconhecida (Münster et al., 2013). Fatores de risco para a regurgitação gastroesofágica, como a presença de doença respiratória, foram identificados tanto em humanos (El-Serag et al., 2014) quanto em cães (Ovbey et al., 2014).

Há muito interesse nessa afecção devido à frequente ocorrência (quase 40\%) na população dos países altamente industrializados (Piatkowski et al., 2014). A regurgitação gastroesofágica é de tamanha importância que é considerada fator de alto risco para o desenvolvimento de adenocarcinoma esofágico, bem como de baixo risco para a ocorrência de adenocarcinoma da cárdia em humanos (Lagergren et al., 1999).

Procedimentos associados com aumento da ocorrência de pneumonia aspirativa por regurgitação em cães incluem laparotomia, cirurgia do trato respiratório superior, neurocirurgia, toracotomia e endoscopia. Cirurgia ortopédica, oftalmológica, procedimentos odontológicos, ressonância magnética, tomografia computadorizada, cistoscopia, traqueoscopia e orquiectomia não foram relacionados ao desenvolvimento de pneumonia aspirativa por regurgitação (Ovbey et al., 2014). Na rotina dos autores deste trabalho, vivenciou-se o caso de uma cadela que foi acometida por estenose esofágica após a realização de ovário-histerectomia videoassistida eletiva, durante a qual apresentou regurgitação no transoperatório (Oliveira et al., 2013), além de outras situações esporádicas de regurgitação intensa observadas durante videocirurgias. Silva et al. (2010) descrevem o caso de outra cadela que foi acometida por estenose esofágica após a realização de ovário-histerectomia por laparotomia, durante a qual houve regurgitação intensa.

$\mathrm{Na}$ cirurgia veterinária tanto a ováriohisterectomia quanto a ovariectomia são procedimentos cirúrgicos utilizados na esterilização eletiva de cadelas e gatas, com o objetivo de impedir a reprodução ou tratar várias afecções do trato reprodutivo, dentre elas: cistos ovarianos, piometra, torção e prolapso uterinos (Schiochet et al., 2007). Sua utilização ainda elimina o risco de falsa gestação (Martins e Lopes, 2005) e reduz a incidência de neoplasias mamárias (Fonseca e Daleck, 2000), além de ser coadjuvante no tratamento de epilepsia (Stone et al., 1998) e diabetes melito (Pöppl, 2012). Intervenções minimamente invasivas têm sido empregadas como alternativa à ováriohisterectomia realizada por laparotomia (Malm et al., 2004). Essas apresentam vantagens quando comparadas com cirurgias convencionais, como diminuição do sangramento (Brun, 1999), do tempo de convalescença, da dor no período pósoperatório e breve retorno às atividades (Basso et al., 2014).

A problemática das complicações do refluxo gastroesofágico perioperatório, a raridade de informações sobre a real ocorrência dele durante os procedimentos cirúrgicos em pequenos animais e a escassez de relatos comparativos entre procedimentos convencionais (celiotomia) e videocirúrgicos em relação ao refluxo motivaram a escolha desse tema. Objetivou-se, com este trabalho, obter esclarecimentos sobre a real influência do tipo de abordagem operatória para OVH com a ocorrência de refluxo gastroesofágico transoperatório.

\section{MATERIAL E MÉTODOS}

Foram atendidas 20 cadelas, adultas, hígidas, sem histórico de êmese, pesando em média $13,9 \pm 4,1 \mathrm{~kg}$ com indicação de ováriohisterectomia (OVH) eletiva. Após aptidão confirmada por exame clínico e laboratorial 
(hemograma e bioquímica sérica), foram aleatoriamente separadas em dois grupos de igual número. Um foi submetido à $\mathrm{OVH}$ convencional, ou seja, realizada por meio de celiotomia (GI), e outro à $\mathrm{OVH}$ videoassistida por dois portais (GII), técnica na qual os portais de acesso são posicionados na linha média e há lateralização do animal para decúbito direito e esquerdo para apreensão dos ovários no transoperatório e uma etapa convencional para hemostasia dos vasos uterinos e remoção dos úteros e ovários. As cadelas de ambos os grupos tiveram o mesmo período de jejum (com internação hospitalar) 12 horas de privação alimentar e oito horas de privação hídrica - e ao mesmo protocolo anestésico - acepromazina $\left(0,05 \mathrm{mg} \cdot \mathrm{kg}^{-1}\right)$ e morfina $\left(0,5 \mathrm{mg} \cdot \mathrm{kg}^{-1}\right)$ via intramuscular como pré-medicação, seguido de propofol $\left(4 \mathrm{mg} \cdot \mathrm{kg}^{-1}\right)$ para indução e isoflurano, vaporizado em oxigênio a $100 \%$, para manutenção anestésica por meio de intubação orotraqueal.

O endoscópio flexível de 9,8mm foi introduzido no esôfago das cadelas até aproximadamente a altura do nono espaço intercostal - mensurado externamente, a fim de observar diretamente a transição esofagogástrica durante o procedimento. Esse posicionamento foi realizado simultaneamente à colocação dos campos operatórios. A ocorrência de refluxo gastroesofágico foi sendo monitorada e correlacionada com os momentos transoperatórios. A insuflação da cavidade, no caso das $\mathrm{OVH}$ videoassistidas, deu-se com $\mathrm{CO}_{2}$ medicinal até se obter a pressão intra-abdominal de $12 \mathrm{mmHg}$, à velocidade de $1,5 \mathrm{~L} / \mathrm{min}$.

O grau de refluxo gastroesofágico foi classificado conforme a legenda: R0 - sem refluxo, esôfago limpo; R1 - sem refluxo, com bolhas desde o início da esofagoscopia, indicativo de peristaltismo pré-operatório; R2 sem refluxo, com formação de bolhas no transoperatório, o que indica um aumento de peristaltismo transoperatório; R3 - refluxo discreto (alcançando a altura do terço distal do esôfago); R4 - refluxo discreto com prolapso de mucosa gástrica para o esôfago; R5 - refluxo moderado (atingindo terço médio do esôfago); R6 - refluxo moderado com prolapso de mucosa gástrica; R7 - refluxo grave (atingindo terço cranial do esôfago) e R8 - refluxo grave com prolapso de mucosa gástrica. Essa escala foi proposta pelos autores do presente trabalho especialmente para o projeto que deu origem a este trabalho e com o intuito de contribuir como método de avaliação endoscópica de intensidade de refluxo.

Este trabalho foi aprovado pelo Comitê de Ética no Uso de Animais (Ceua) - UFSM, sob o parecer 136/2014. Data de aprovação: $18 / 12 / 2014$.

\section{RESULTADOS E DISCUSSÃO}

Rotineiramente, para pequenos animais, é solicitado jejum alimentar de 12 horas, cogitando-se alterações conforme idade do paciente e procedimento cirúrgico a ser realizado (Muir, 2007). A fim de se buscarem animais nos quais o conteúdo alimentar não influenciasse nas avaliações, para o presente trabalho foram selecionadas cadelas sem histórico de êmese, que fossem alimentadas 12 horas anteriores ao procedimento, quando eram internadas no canil, para garantir a realização do jejum. Dessa forma, descartou-se a possibilidade de acesso à alimentação líquida ou sólida após o período predeterminado, já que tal variável poderia interferir diretamente nos resultados de vômitos e/ou regurgitações. Entre todas as cadelas, 12 (60\%) tiveram êmese após a medicação pré-anestésica. Wilson et al. (2005) observaram que a incidência de êmese após a administração de morfina na pré-medicação aumentou significantemente conforme o aumento na dose desse fármaco associado à acepromazina.

Das 10 cadelas do GI, sete tiveram êmese após a medicação pré-anestésica e três apresentaram refluxo gastroesofágico transoperatório classificado em R3. Duas cadelas manifestaram $\mathrm{R} 1$ e duas manifestaram R2; essas duas classificações não foram consideradas efetivamente refluxo por não se enquadrarem na definição de Norton e Pena (2000), Barczinski e Moraes-Filho (2006), Carvalhaes et al. (2011) e Abrahão Jr. (2014) para tal condição. Nesses casos, considerou-se que a presença de bolhas ou espuma já no início da endoscopia indicou peristaltismo esofágico saliente no préoperatório, e a formação de bolhas ou espuma durante o procedimento indicou peristaltismo transoperatório, sendo talvez uma fase prévia do refluxo propriamente dito. 
Das 10 cadelas do GII, cinco manifestaram êmese após a medicação pré-anestésica e todas manifestaram refluxo gastroesofágico transoperatório. Três animais tiveram o refluxo classificado em grau R4, quatro em grau R5, um em grau R6, um em R7 e um em R8. Assim, a êmese pré-operatória não se apresentou como condição determinante de refluxo gastroesofágico transoperatório, visto que dessas três cadelas do GI, duas manifestaram o primeiro sinal e outras cinco que tiveram êmese não tiveram refluxo. Esses achados vem ao encontro dos de Wilson et al. (2005), que igualmente não observaram relação entre essas duas condições clínicas. Sabe-se, ainda, que a morfina é um dos fármacos já descritos como precipitadores do refluxo gastroesofágico (Barczinski e MoraesFilho, 2006), condição também comprovada no presente estudo.

A Fig. 1 representa a distribuição das cadelas conforme a abordagem para realização da $\mathrm{OVH}$ e os graus de refluxo gastroesofágico transoperatório, ficando evidente que, enquanto as submetidas à $\mathrm{OVH}$ convencional tiveram, no máximo, refluxos classificados em R3, as cadelas submetidas à $\mathrm{OVH}$ videoassistida tiveram, no mínimo, refluxos classificados em R4. A Fig, 2 exemplifica diferentes graus de refluxos observados no transoperatório.

Procedimentos que já foram relacionados a refluxo gastroesofágico constam de $\mathrm{OVH}$ convencional (Silva et al., 2010), OVH videoassistida (Oliveira et al., 2013) e intervenções ortopédicas em membros posteriores (Wilson et al., 2005). Eventos que já foram relacionados à pneumonia aspirativa em consequência do refluxo incluem laparotomia, cirurgia do sistema respiratório superior, neurocirurgia, toracotomia e endoscopia (Ovbey et al., 2014), demonstrando, portanto, a necessidade de se investigarem outras causas de refluxo gastroesofágico que não somente $\mathrm{o}$ pneumoperitôneo, as alterações de decúbito e as demais circunstâncias inerentes à prática da $\mathrm{OVH}$ videoassistida com dois portais.

Os momentos em que ocorreram (ou começaram a ocorrer) os refluxos gastroesofágicos nas cadelas submetidas à $\mathrm{OVH}$ convencional coincidiram, tratando-se da etapa de manipulação para exteriorização do primeiro ovário - o direito. A hipótese dos autores é a de que isso possa ter acontecido na cirurgia convencional pelo estímulo doloroso associado à ruptura do ligamento suspensor. Por outro lado, na $\mathrm{OVH}$ videocirúrgica, o suspensor é muito pouco manipulado e não é rompido sob tração, tal como feito na técnica convencional. Para o grupo da videocirurgia, acredita-se que a lateralização da paciente associada ao pneumoperitôneo possa precipitar o refluxo. Porém, não há, na literatura consultada, comentários sobre isso.

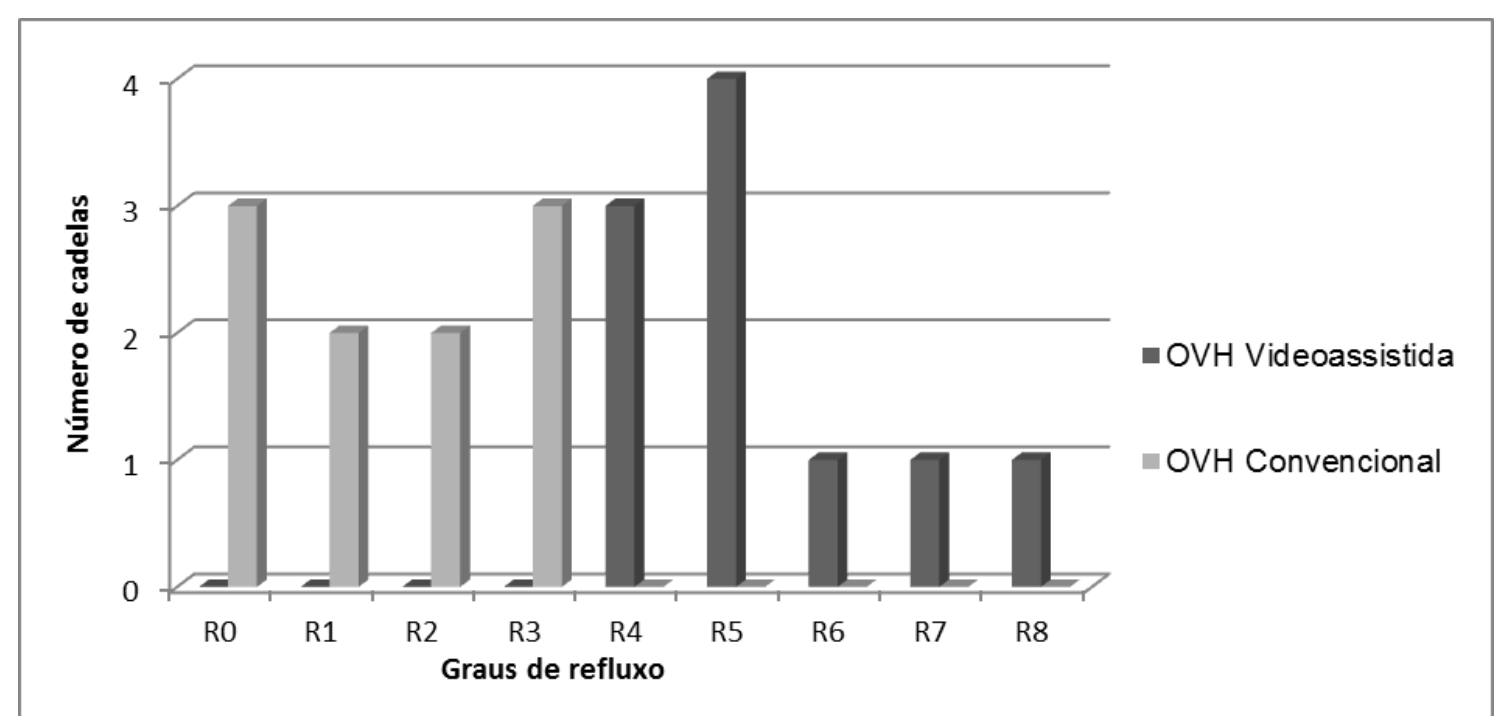

Figura 1. Gráfico representativo do número de cadelas em relação aos graus de refluxo e ao tipo de abordagem cirúrgica. 


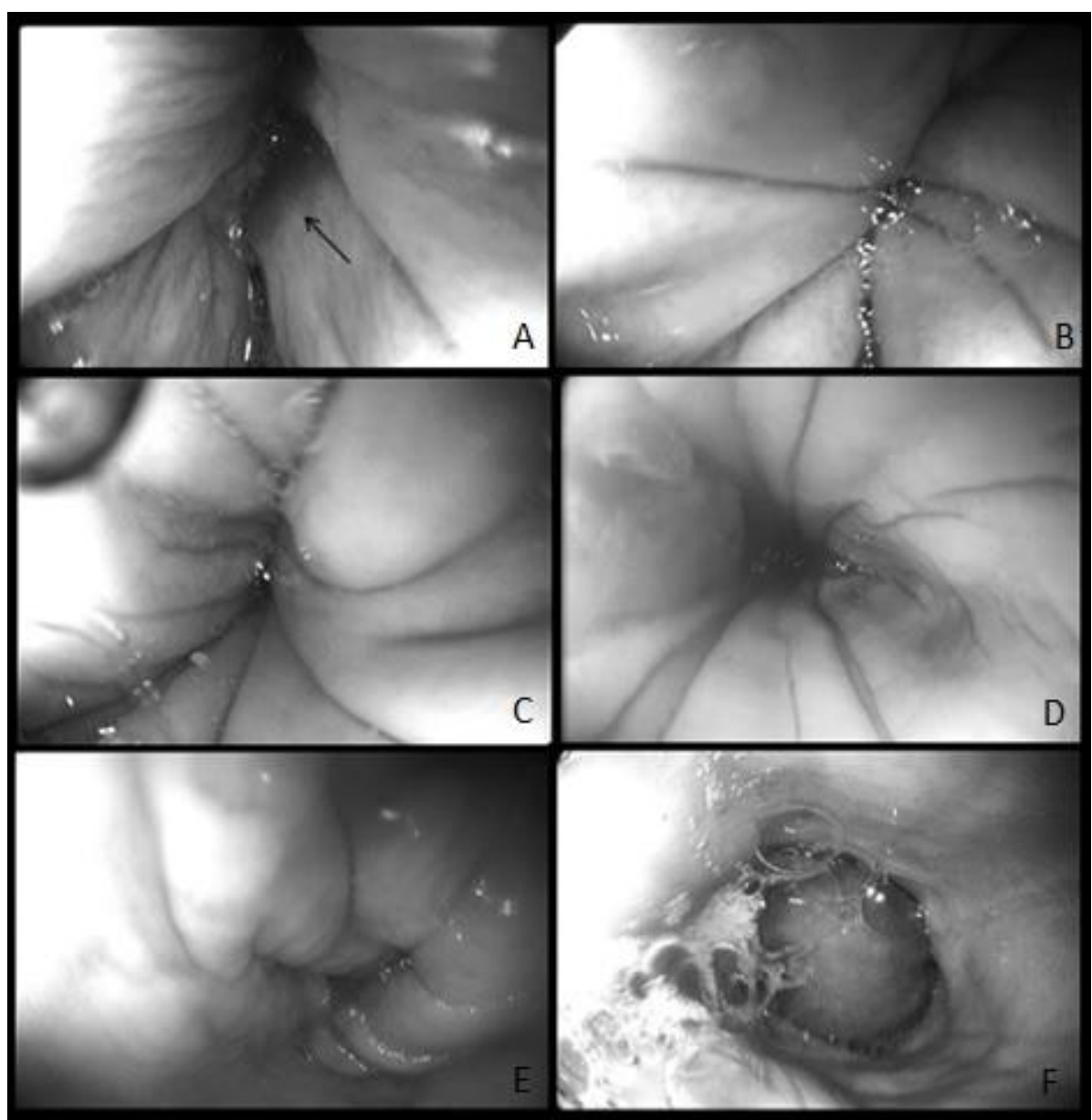

Figura 2. Imagens endoscópicas das cadelas submetidas à $\mathrm{OVH}$ convencional e à $\mathrm{OVH}$ videoassistida. A) esôfago com bolhas desde o início do procedimento; atente-se para o detalhe da junção esôfago-gástrica (seta). B) formação de bolhas ao longo do procedimento, evento graduado como R2. C) refluxo gastroesofágico discreto (R3), líquido límpido acumulado no terço caudal do esôfago. D) refluxo gastroesofágico moderado, pois o líquido alcançou o terço médio do esôfago (R5). E) imagem evidenciando início de prolapso de mucosa gástrica. F) situação de refluxo moderado (ao recuo do endoscópio, pode-se averiguar que o conteúdo atingiu o terço médio do esôfago) com prolapso de mucosa gástrica (R6).

Os momentos em que ocorreram (ou começaram a ocorrer) os refluxos nas cadelas submetidas à $\mathrm{OVH}$ videoassistida variaram. Sete manifestaram o evento após a insuflação da cavidade, sendo duas em grau R3, três em grau R4 e duas em grau R5. Os outros três animais começaram a ter refluxo gastroesofágico a partir da primeira alteração de decúbito para manipulação do complexo arteriovenoso ovariano (CAVO), sendo nos graus R3, R4 e R5 cada uma e sendo esses os refluxos mais graves que apresentaram durante o procedimento. Nas cadelas que iniciaram os refluxos após a insuflação cavitária, houve situações mais graves durante as alterações de decúbito e manipulação dos CAVOs, inclusive chegando a R8 em uma delas.
A insuflação da cavidade com o pneumoperitôneo de $12 \mathrm{mmHg}$ na velocidade de $1,5 \mathrm{~L} / \mathrm{min}$, bem como as alterações de decúbito transoperatórias, precipita o refluxo gastroesofágico em animais submetidos ao protocolo anestésico descrito. Acredita-se que a influência do pneumoperitôneo associada ao uso da morfina seja maior do que a influência da morfina isoladamente na pré-medicação, visto que das cadelas submetidas à $\mathrm{OVH}$ convencional $30 \%$ manifestaram refluxo gastroesofágico 
transoperatório contra $100 \%$ das cadelas submetidas à $\mathrm{OVH}$ videoassistida. Os autores cogitam ainda que a velocidade de insuflação da cavidade possa ter influência sobre o refluxo gastroesofágico.

Todos os animais tiveram os refluxos aspirados como preconizado por Hall (2015). Uma cadela teve refluxo com conteúdo alimentar volumoso; esta teve o esôfago lavado e aspirado após o procedimento. Várias podem ser as consequências do refluxo gastroesofágico, dentre elas: azia, esofagite (Münster et al., 2013), estenose esofágica (Adamama-Moraitou et al., 2002; Camargo et al., 2003; Vlasin et al., 2004; Corgozinho et al., 2006; Favarato et al., 2010; Silva et al., 2010; Lam et al., 2013; Oliveira et al., 2013; Ovbey et al., 2014) e pneumonia aspirativa (Adamama-Moraitou et al., 2002; Favarato et al., 2010; Ovbey et al., 2014). Dessa forma, os autores indicam sempre o manejo clínico de pacientes nos quais se constata essa alteração, incluindo atualmente o uso de omeprazol, independentemente do grau de refluxo ocorrido.

Uma manobra comum para evitar aspiração de conteúdo gástrico no período transanestésico é a remoção do tubo endotraqueal com o cuff parcialmente repleto, tracionando para o exterior da traqueia eventuais produtos de refluxo (D'Haese et al., 2013). Os animais do presente estudo não necessitaram da realização dessa manobra, visto que a aspiração endoscópica dos produtos de refluxo foi efetiva.

Na cadela que teve refluxo em grau $\mathrm{R} 8$, houve a entrada não planejada do endoscópio no estômago. Apesar de a entrada ter sido em um primeiro momento, no início da colocação dos campos cirúrgicos, e o refluxo grave ter sido na última alteração de decúbito, ao final do procedimento, não se descarta a possibilidade de interferência pela abertura da cárdia. Em uma cadela submetida à OVH convencional, também ocorreu a entrada não planejada do endoscópio no estômago no momento de posicionamento desse, simultaneamente à colocação dos campos operatórios. Esse animal não manifestou nenhum grau de refluxo.

Não foi realizada pHmetria do conteúdo que atingiu o esôfago das cadelas, como realizado por Wilson et al. (2005), porém aquelas que manifestaram refluxo tiveram a prescrição pósoperatória de omeprazol $\left(1 \mathrm{mg} \cdot \mathrm{kg}^{-1}\right)$ via oral, a cada $24 \mathrm{~h}$, durante cinco dias. Esse tratamento consiste em reduzir a acidez gástrica, prevenir refluxos subsequentes e proteger a mucosa esofágica (Nelson e Couto, 2010). Nenhum animal deste trabalho teve qualquer complicação pós-operatória referente a sinais gastrointestinais ou complicações cicatriciais num período de, no mínimo, 21 e, no máximo, 98 dias após os procedimentos cirúrgico e endoscópico.

\section{CONCLUSÃO}

Considerando-se o protocolo analgésico utilizado e as condições do pneumoperitôneo obtido, as cirurgias $\mathrm{OVH}$ videoassistidas promoveram mais refluxo gastroesofágico transoperatório que as cirurgias por abordagem convencional, sendo os refluxos associados ao acesso minimamente invasivo de maior gravidade. Alguns fatores foram atribuídos como contributivos para o refluxo, como a administração de morfina na prémedicação anestésica, a criação e manutenção do pneumoperitôneo e as alterações de decúbito durante o procedimento.

\section{REFERÊNCIAS}

ABRAHÃO JR, L.J. Doença do refluxo gastroesofágico. J. Bras. Med., v.102, p.31-36, 2014.

ADAMAMA-MORAITOU, K.K.; RALLIS, T.S.; PRASSINOS, N.N.; GALATOS, A.D. Benign esophageal stricture in the dog and cat: a retrospective study of 20 cases. Can. J. Vet. Res., v.66, p.55-59, 2002.

BARCZINSKI, T.; MORAES-FILHO, J.P.P. Doença do refluxo gastroesofágico na mulher. Rev. Bras. Med., v.63, p.160-168, 2006.

BASSO, P.C.; RAISER, A.G.; BRUN, M.V. et al. Biomarcadores inflamatórios e indicadores de estresse oxidativo em cadelas submetidas à ovariossalpingohisterectomia convencional, por NOTES híbrida e NOTES total. Ciênc. Rural, v.44, p.884-890, 2014.

BRUN, M.V. Ovário-histerectomia em caninos por cirurgia laparoscópica. 1999. $181 \mathrm{f}$. Dissertação (Mestrado em Ciências Veterinárias) - Programa de Pós-graduação em Ciências Veterinárias, Universidade Federal do Rio Grande do Sul, Porto Alegre, RS. 
CAMARGO, M.A.; LOPES. L.R.; GRANGEIA, T.A.G. et al. O uso de corticosteróides após dilatação esofágica em pacientes portadores de estenose por substâncias corrosivas - estudo prospectivo, randomizado e duplo-cego. Rev. Assoc. Med. Bras., v.49, p.286-292, 2003.

CARVALHAES, A.; FERRARI JR., A.P.; MAGALHÃES, A.F. et al. Doença do refluxo gastroesofágico: diagnóstico. Rev. Assoc. Med. Bras., v.57, p.499-507, 2011.

CORGOZINHO, K.B.; NEVES, A.; BELCHIOR, C. et al. Uso de triancinolona local em uma gata com estenose esofágica. Acta Sci. Vet., v.34, p.175-178, 2006.

D'HAESE, J.; KEUKELEIRE, T.; REMORI, I. et al. Assessment of intraoperative microaspiration: does a modified cuff shape improve sealing? Acta Anaesthesiol. Scand., v.57, p.873-880, 2013.

EL-SERAG, H.B.; SWEET, S.; WINCHESTER, C.C.; DENT, J. Update on the epidemiology of gastro-oesophageal reflux disease: a systematic review. Gut, v.63, p.871-880, 2014.

FAVARATO, E.S.; SOUZA, M.M.; COSTA, P.R.S. Refluxo gastroesofágico em cães anestesiados: fisiopatologia, clínica, diagnóstico e terapêutica. Ciênc. Rural, v.40, p.2427-2434, 2010.

FONSECA, C.S.; DALECK, C.R. Neoplasias mamárias em cadelas: influência hormonal e efeitos da ováriohisterectomia como terapia adjuvante. Ciênc. Rural, v.30, p.731-735, 2000.

HALL, E. Endoscopy of the gastrointestinal tract in dogs and cats. In Pract., v.37, p.155-168, 2015 .

JANDA, M.; SCHEEREN, T.W. Management of pulmonary aspiration. Best Pract. Res. Clin. Anaesthesiol., v.20, p.409-427, 2006.

LAGERGREN, J.; BERGSTRÖM, R.; LINDGREN, A.; NYRÉN, O. Symptomatic gastroesophageal reflux as a risk factor for esophageal adenocarcinoma. N. Engl. J. Med., v.340, p.825-831, 1999.

LAM, N.; WEISSE, C.; BERENT, A. et al. Esophageal stenting for treatment of refractory benign esophageal strictures in dogs. J. Vet. Intern. Med., v.27, p.1064-1070, 2013.
MALM, C.; SARAVASSI-ROCHA, V.A.; GHELLER, H.P. et al. Ovário-histerectomia: estudo experimental comparativo entre as abordagens laparoscópica e aberta na espécie canina - intraoperatória - I. Arq. Bras. Med. Vet. Zootec., v.56, p.457-466, 2004.

MARTINS, L.R.; LOPES, M.D. Pseudociese canina. Rev. Bras. Reprod. Anim., v.29, p.137141, 2005.

MUIR, W.W. Considerations for general anesthesia. In: TRANQUILI, W.J. et al. Lumb \& Jones'veterinary anesthesia and analgesia. 4.ed. Iowa: Blackwell Publishing, 2007. p.7-30.

MÜNSTER, M.; VIETH, M.; HÖRAUF, A. Evaluation of the quality of endoscopically obtained esophageal biopsies in the dog. Tierärztl. Prax. K., v.41, p.375-382, 2013.

NELSON, R.W.; COUTO, C.G. Doenças da cavidade oral, da faringe e do esôfago. In: Medicina interna de pequenos animais. 4.ed. Rio de Janeiro: Elsevier, 2010. cap.31, p.414-425.

NOGUEIRA, L.C.; CORTOPASSI, S.R.G.; INTELIZANO, T.R.; SOUZA, M.S.B. Efeitos do jejum alimentar pré-cirúrgico sobre a glicemia e o período de recuperação anestésica em cães. Braz. J. Vet. Res. Anim. Sci., v.40, Supl.1, p.2025, 2003.

NORTON, R.C.; PENNA, F.J. Refluxo gastroesofágico. J. Pedriatr., v.76, Supl.2, p.218224, 2000.

OLIVEIRA, M.T.; TRINDADE, A.B.; SOUZA, F.W. et al. Dilatação esofágica endoscópica associada ao uso de triancinolona intramural em cadela com estenose de esôfago após ovariohisterectomia eletiva. Ciênc. Rural, v.43, p.1683-1686, 2013.

OVBEY, D.H.; WILSON, D.V.; BEDNARSKI, R.M. et al. Prevalence and risk factors for canine post-anesthetic aspiration pneumonia (19992009): a multicenter study. Vet. Anaesth. Analg., v.41, p.127-136, 2014.

PIATKOWSKI, J.; JACKOWSKI, M.; SZELIGA, J. Laparoscopic surgery of esophageal hiatus hernia - single center experience. Videosurg. Miniinvasive Techn., v.9, p.13-17, 2014. 
PÖPPL, A.G. Estudos clínicos sobre os fatores de risco e a resistência à insulina na diabetes mellitus em cães. 2012. 215f. Tese (Doutorado em Ciências Veterinárias) - Programa de Pósgraduação em Ciências Veterinárias, Universidade Federal do Rio Grande do Sul, Porto Alegre, RS.

SCHIOCHET, F.; BECK, C.A.C.; STEDILE, R. et al. Ovariectomia laparoscópica em uma gata com ovários remanescentes. Acta Sci. Vet., v.35, p.245-248, 2007.

SILVA, E.C.S.; PINA, F.L.S.; TEIXEIRA, M.W. Diagnóstico e tratamento da estenose esofágica pela via endoscópica em cão: relato de caso. Cienc. Anim. Bras., v.11, p.465-470, 2010.
STONE, E.A. Ovário e útero. In: SLATTER, D. Manual de cirurgia de pequenos animais. 2.ed. São Paulo: Manole, 1998. p.1540-1558.

VLASIN, M.; HUSNIK, R.; FICHTEL, T.; RAUSEROVÁ, L. Acquired esophageal stricture in the dog: a case report. Vet. Med. Czech. J., v.49, p.143-147, 2004.

WILSON, D.V.; EVANS, A.T.; MILLER, R. Effects of preanesthetic administration of morphine on gastroesophageal reflux and regurgitation during anesthesia in dogs. Am. J. Vet. Res., v.66, p.386-390, 2005. 